\title{
An Improved Approach for Visualizing Dynamic Vision Sensor and its Video Denoising
}

\author{
Xuemei Xie ${ }^{1}$, Jiang $\mathrm{Du}^{1}$, Guangming Shi ${ }^{1}$, Hong $\mathrm{Hu}^{2}$, Wang $\mathrm{Li}^{1}$ \\ ${ }^{1}$ School of Artificial Intelligence \\ ${ }^{2}$ School of Electronic Engineering \\ Xidian University,Xi'an, Shaanxi 710071, China \\ xmxie@mail.xidian.edu.cn
}

\begin{abstract}
Dynamic vision sensor (DVS) is an event-based camera capturing the changes of vision with high speed and low storage consumption. To better understand what DVS captures, we need to visualize the events. Existing methods have realized visualization. To optimize the vision experience, this paper proposes a framework to visualize events with rich information, high speed and less noise. Firstly, we propose an improved visualization approach using overlapped events based on human vision system. Secondly, we propose a video denoising method using shared dictionaries. In our experiments, the proposed method realizes the expected purpose on the whole video.
\end{abstract}

\section{CCS Concepts}

- Computing methodologies $\rightarrow$ Artificial intelligence $\rightarrow$ Computer vision $\rightarrow$ Computer vision problems $\rightarrow$ Reconstruction.

\section{Keywords}

Dynamic vision sensor, Visualization,Video denoising,Shared dictionary

\section{INTRODUCTION}

Dynamic vision sensor (DVS) is a kind of event-based camera [1] [2] [3] [4], which works at high speed with little storage consumption. A typical DVS is shown in Figure 1.

DVS captures the scene in the form of events, which does not cater to human vision. With visualization, it can help us

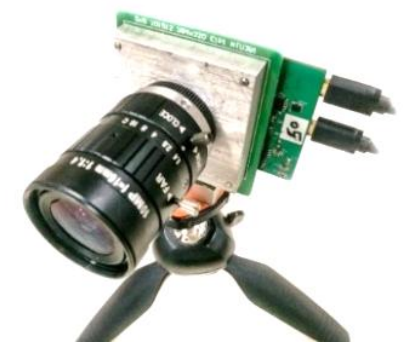

Figure 1. A typical dynamic vision sensor

Permission to make digital or hard copies of all or part of this work for personal or classroom use is granted without fee provided that copies are not made or distributed for profit or commercial advantage and that copies bear this notice and the full citation on the first page. Copyrights for components of this work owned by others than ACM must be honored. Abstracting with credit is permitted. To copy otherwise, or republish, to post on servers or to redistribute to lists, requires prior specific permission and/or a fee. Request permissions from Permissions@ acm.org. ICVIP 2017, December 27-29, 2017, Singapore, Singapore (C) 2017 Association for Computing Machinery. ACM ISBN 978-1-4503-5383-0/17/12 ..\$15.00 DOI: https://doi.org/10.1145/3177404.3177411

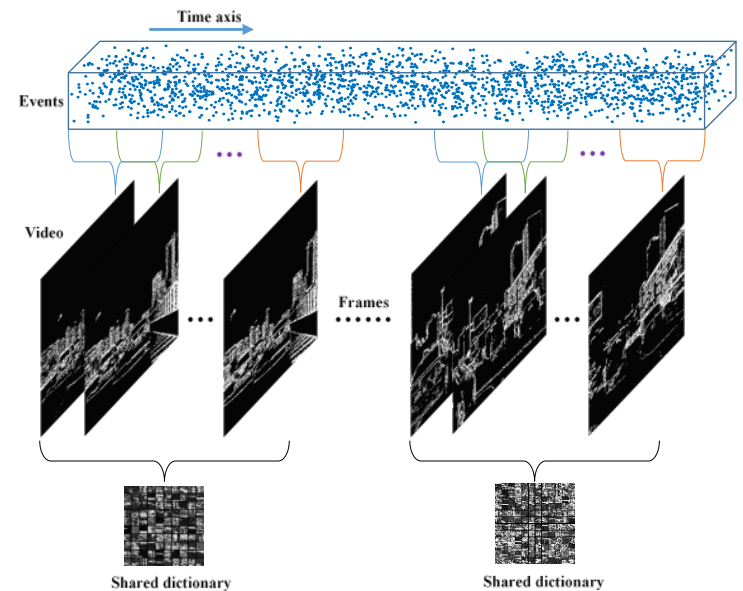

Figure 2. The proposed framework of events being converted to video, where the events are converted to frames overlappedly, and the video denoising is applied with shared dictionary.

understand what DVS captures. Guo, Ding, and Chen [1] proposed an approach for visualizing what DVS captures. What's more, this approach can realize visualization at high frame rate and the frame rate is adjustable. To reach the high rate, it will reduce the information in one frame. In this paper, we propose an improved visualization method. This method can visualize DVS with both high speed and rich information. The framework for realizing this visualization is in Figure 2. In our proposed framework, the events are converted to video and they are overlapped to increase the number of events in each frame. The shared dictionaries, which represent the frames, are used group by group to reduce time consumption.

With the increase of information, noise will also become heavy. Since the events DVS captures are in the form of binary stream, impulse noise [5] is likely to be adulterated while transferring digital signal. Other noise can also jointly damage our visualized video's quality, including Gaussian white noise [6] caused by MOSFET or photodiodes, FPN (fixed pattern noise) [7] caused by different light sensitivity of photodiodes in the sensor, 1/f noise [8] and so on. Consider the noise above that affects the vision experience of DVS visualization. In this paper, our framework in Figure 2. also deals with the noise of video. We remove the noise with shared dictionary. Since the feature of DVS video changes slightly with frames, shared dictionary works well on DVS video. Our method realizes clear structure of objects on the video.

The structure of this paper is as follows. Section 2 briefly introduces the related work. The improved method of visualization using overlapped events is illustrated in Section 3. Some tricks about denoising are presented in Section 4. Section 5 
shows the experiments and Section 6 draws a conclusion of this paper.

\section{RELATED WORK}

\subsection{Existing methods of DVS visualization}

DVS captures the scene in the form of events. To understand what is captured, there are two existing approaches [1] to visualize the events. One is full-frame and another is picture-on-demand that reconstructed events received within a certain time slice. In the first method, a full-frame picture is generated on the condition where every pixel generates at least one event. It requires a "Force-fire" control signal. The full-frame pictures look just the same as images captured by traditional gray-scale cameras [9]. In the second method, a picture-on-demand image is generated using just the events within a certain time slice. This time slice can be controlled with GUI. This method does not need a "Force-fire" signal and can start visualizing at any time. It accumulates the changed pixels to form an image.

There are three categories of events: row events, column events and special events. Row events carry row address and the activation time for the pixels on that row. Column events follow right after a row event. They have the brightness information for pixels. The timing information can be retrieved from the preceding row event. Special events represent the end of a time slice. After this event, timer count resets to zero. The length of the time slice is configurable.

\subsection{Existing methods of video denoising}

Currently, there are many denoising methods. The technique based on multi-dimensional signal processing approach is proposed [10], which can obtain a good enhancement. The video denoising algorithm based on patch is proposed [11], which is capable of removing serious mixed noise from the video data. Besides, a video denoising method, combining spatial and temporal filters [12], is proposed. BM3D denoising algorithm [13] based on the block-matching and $3 \mathrm{D}$ transform domain filtering is evaluated, resulting the brilliant performance. However, the noise from DVS video has unique features, where the noise and structure of objects have similar property, both consisting of discrete points. It is hard to apply traditional methods to remove this kind of noise. In this paper, we present a new approach with shared dictionary, which gives an excellent performance on denoising the DVS video.

\section{IMPROVED METHOD OF VISUALIZING DVS - OVERLAPPED EVENTS}

The existing method has managed visualization of DVS. This paper attempts to improve the visual feeling. The problem to be solved here is to realize both high frame rate and rich information in one frame. This section will address this problem in detail.

Accumulating events in a short time slice will cause a weak structure of objects in one frame of DVS video, while in a long time slice will lose its advantage of high speed.

Considering the relationship between time slice and frame rate, we give an example for illustration in Figure 3. In Figure 3(a), the time slice is long, but its frame rate is low. In Figure 3(b) the frame rate is high, but one frame contains poor information.

When we human beings see the world, everything will be left in our sight for about 0.1 second. Inspired by persistence of vision [14], we visualize the events with overlapping method. For example, we can convert events from 0 millisecond to 30 milliseconds for frame 0 . Then we convert events from 10

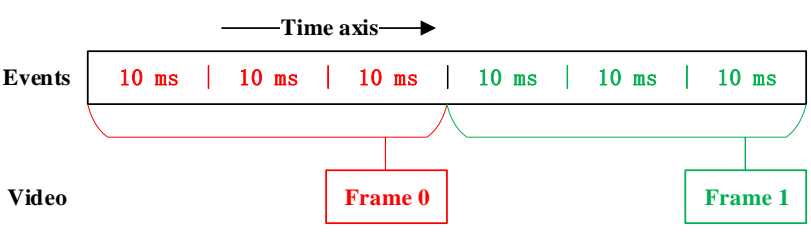

(a)

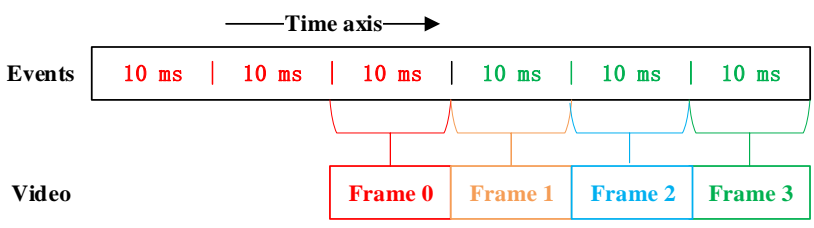

(b)

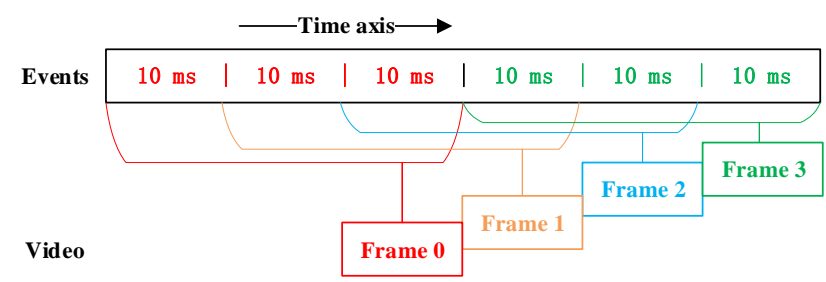

(c)

Figure 3. Time slice of frames and its relationship among frames with different methods. (a) existing method aiming at rich information in one frame. (b) existing method aiming at high frame rate. (c) proposed method realizing both high frame rate and rich information in one frame

milliseconds to 40 milliseconds for frame 1 . In this case, each frame contains the information of events within 30 milliseconds of time slice, where information is rich enough, but this framework can maintain up to 100 frames per second. This description is shown in Figure 3(c).

Different time slice contains different amount of events in each frame. To measure the time slice accurately, we can use special events. However, there may be some risks that different frames contain different amount of events. Instead, we just use the number of normal events to segment the frames. This can make the video more smooth. In this case, the time slice of one frame can be indirectly calculated with the number of events.

Here we take a 6-second-long video as an example. When

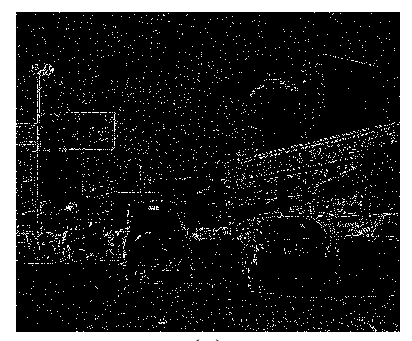

(a)

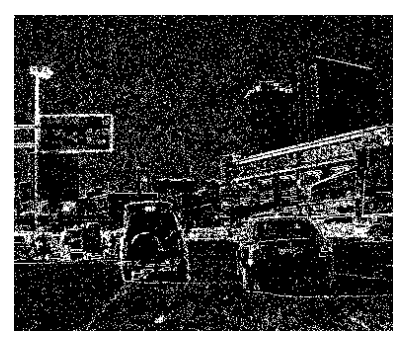

(b)
Figure 4. Frames with existing method and proposed method at the same scene. (a) existing method, and (b) proposed method. 
Table 1. Comparison of Parameters in some Terms using Different Methods

\begin{tabular}{ccccc}
\hline Images & $\begin{array}{c}\text { Visualization } \\
\text { method }\end{array}$ & $\begin{array}{c}\text { Frame } \\
\text { rate }\end{array}$ & $\begin{array}{c}\text { Number } \\
\text { of events }\end{array}$ & $\begin{array}{c}\text { Time slice } \\
\text { (milliseconds) }\end{array}$ \\
\hline \hline Image (a) & Existing & 122 & 6000 & 8.2 \\
Image (b) & Proposed & $\mathbf{1 2 2}$ & $\mathbf{3 0 0 0 0}$ & $\mathbf{4 0 . 8}$ \\
\hline
\end{tabular}

Images mentioned in this table are in Figure 4

reaching the same frame rate as existing visualization method, our method performs better than existing method. Both cases are shown in Figure 4, where Figure 4(a) and (b) are given for existing and proposed, respectively. It is obvious that the proposed method of visualization shows clear structure information of objects, maintaining high frame rate at the same time. From Table1, we can see frame rate, number of events (per frame) and time slice.

\section{VIDEO DENOISING BASED ON SHARED DICTIONARY}

After visualizing the DVS events, we find that the video contains a lot of noise. In this section, we propose a video denoising approach with shared dictionary based on K-SVD algorithm [15].

Single DVS image K-SVD denoising [16] requires training a dictionary for each new frame. However, it takes a lot of time to train dictionaries for each frame. To reduce time consumption of training, we use a shared dictionary [16] [17] based on K-SVD to denoise different key frames. All of key frames can cover the whole time axis without overlapping. Furthermore, the shared dictionary contains the universal features of adjacent key frames of video. Then, we use the denoising result of key frames to remove the noise of the events, and finally visualize the events again without noise.

\subsection{Training a Shared Dictionary}

In the training phase, we firstly get key frames of the video. Then, we apply single image denoising method to some of these frames. After that, we use the denoised images to train a dictionary, which has universal features of adjacent frames in the video and contains nearly no noise. The detailed training phase of K-SVD is as follows.

We firstly initialize the dictionary with random value uniformly distributed. Then, we solve an optimization problem in (1). The noisy image $\mathbf{Y}$ can be expressed with weighted sum of $\mathbf{D}$ with the coefficientX.

$$
\min _{\mathbf{D}, \mathbf{X}}\left\{\|\mathbf{Y}-\mathbf{D X}\|_{\mathrm{F}}^{2}\right\} \text { s.t. } \forall i,\left\|\boldsymbol{x}_{i}\right\| \leq \mathrm{T}_{0},
$$

where $\boldsymbol{x}_{i}$ is $i$-th element of $\mathbf{X}$. $\mathrm{T}_{0}$ is the threshold to restrict the maximum sparsity of $\boldsymbol{x}_{i}$.

Then, we update the dictionary $\mathbf{D}$. The column of $\mathbf{D}$ is optimized one by one. When we solve the $i$-th column, we fix other $i$ 1columns of $\mathbf{D}$ and only update the $i$-th column. The matrix of error is calculated,

$$
\boldsymbol{E}_{k}=\mathbf{Y}-\sum_{i \neq k} \boldsymbol{d}_{i} \boldsymbol{x}_{T}^{K}
$$

where $\boldsymbol{d}_{i}$ is the $i$-th atom in the dictionary. $\boldsymbol{x}_{T}^{K}$ is the coefficient of the corresponding atoms. Singular value decomposition is used here to process

$$
\boldsymbol{E}_{k}^{R}=\mathbf{U} \Delta \mathbf{V}^{\mathrm{T}}
$$

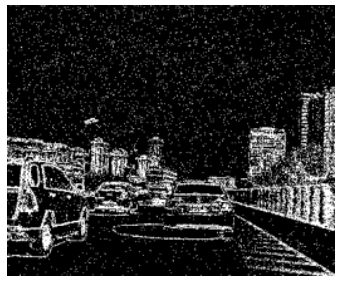

(a)

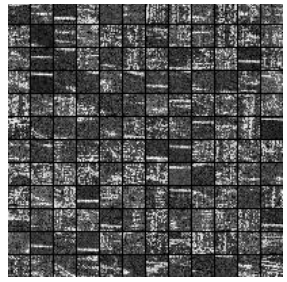

(b)
Figure 5. DVS frame and the dictionary learned from it. (a) DVS frame and (b) its corresponding learned dictionary.

where $\boldsymbol{E}_{k}^{R}$ are columns that corresponds to atoms in the dictionary. The column $\boldsymbol{d}_{i}$ is updated with the first column of matrix $\mathbf{U}$. The vector of coefficient is updated with the first column of Vmultiplies $\Delta(1,1)$. We iterate the above steps from (2) to (3) for a certain times to update both the dictionary and the coefficients. Finally, the reconstructed image can be obtained,

$$
\widehat{\mathbf{Y}}=\mathbf{D X} \text {. }
$$

As a result, the denoised image $\widehat{\mathbf{Y}}$ is obtained.

To explicitly explain this problem, we give an example usingFigure 5(a) as the input image. The dictionary in Figure 5(b) is learned by using K-SVD algorithm.

\subsection{Denoising the Video}

In the denoising phase, we use the shared dictionary to perform denoising on the key frames. To further reduce time consumption, we do not reconstruct all the denoised frames of the video. It can be noticed that the frames of the video is converted from overlapped events. It means the adjacent frames containing almost the same noise. Thus, we do not need the redundant computation. We pick the key frames in the video, which contains the information of all the events without overlapping. Then, we just apply the denoising algorithm with the shared dictionaries to those key frames. After that, we get the position of the noise from the denoising results. According to this, we can go back to events and delete the corresponding events of noise. The denoised events are visualized again with overlapped frames, and finally the video will not contain the removed noise. By avoiding the repeatedly

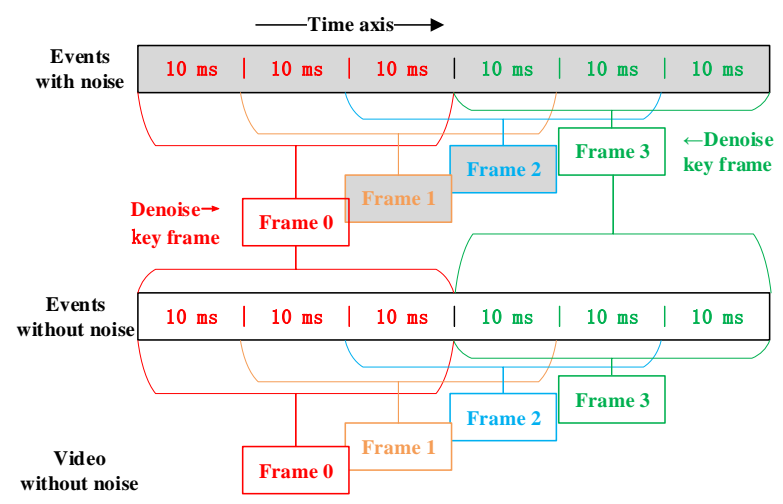

Figure 6. The illustration of frames and time slice in the case of denoising. Firstly we apply denoising algorithm to those key frames with the shared dictionary. Then we go back to events and delete the corresponding events of noise. After that, the denoised events can visualize again with overlapped frame. Finally we get the denoised video. 


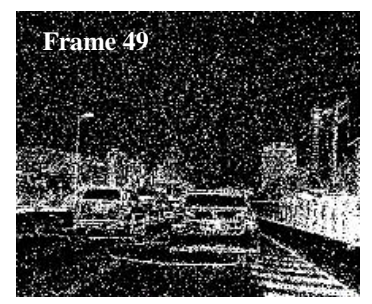

(a)

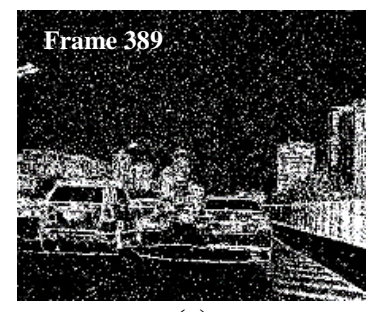

(c)

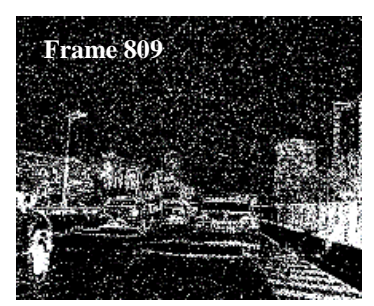

(e)

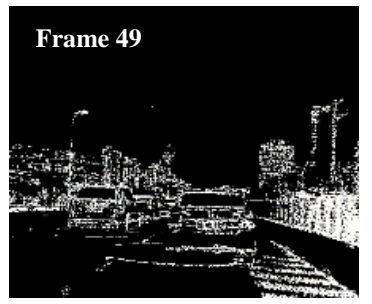

(b)

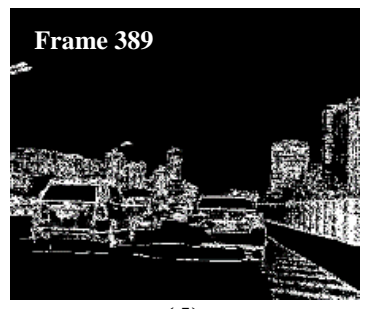

(d)

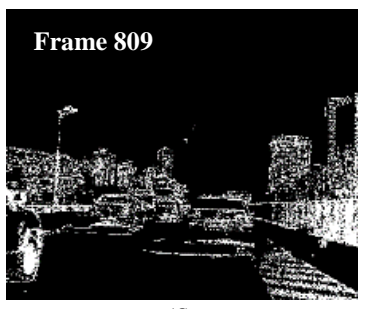

(f)
Figure 7. Results of DVS video denoising. The video contains 840 frames within 10 seconds, with frame rate of 84 fps. (a), (c), (e) noisy frames from the video, and (b), (d), (f) denoised frames corresponding to different frame index.

denoising phase on the same noise, time consumption is greatly reduced. The process of denoising phase is shown in Figure 6.

We will apply such training and denoising process to a real DVS video and the experiment is shown in Section 5.

\section{EXPERIMENT RESULTS}

In this section, we conduct DVS visualization and video denoising experiments on both a fixed DVS and a moving DVS.

The DVS is bought from VIRTUS IC Design Centre of Excellence in Nanyang Technological University [1]. Its device type is CeleX XEM 3010 and serial number is 1540 . We run the visualization program on Microsoft Visual Studio 2013 using C++, and we use the K-SVD toolbox for training and OMP toolbox for optimization on MATLAB R2017a platform in the denoising phase. Our computer is equipped with Intel Core i7-5930K CPU with frequency of $3.5 \mathrm{GHz}$, NVIDIA GeForce GTX $1080 \mathrm{GPU}$, 128GB RAM, and the framework runs on Windows 7 64-bit operating system.

We perform this experiment on a dataset of events within 10 seconds and the visualized video contains 840 frames, where frame rate is at 84 frames per second. The result of the experiment is shown in Figure 7. The parameters are set in Table2.

In our experiment, the block size should be set at least 8, otherwise the block will not contain enough size for structure of the objects when using K-SVD algorithm to learn. But too big
Table 2. Denoising Parameters in the Experiment

\begin{tabular}{cc}
\hline Parameters & Value \\
\hline \hline Noise standard deviation & 0.16 \\
Block size & 10 \\
Number of atoms & 100 \\
Iteration time & 30 \\
\hline
\end{tabular}

block size will rapidly increase time consumption. For example, on our computer, using block size of 10 costs about 2 seconds for denoising a frame, but using that of 16 will costs about 1 minute. The number of atoms is set according to the sparsity of the input signal. Noise standard deviation is set according to the energy of the noise in a frame. In a continuous DVS video, difference between frames is not distinct. Thus, we can find a suitable value for the whole video. We set the iteration time to 30, which can ensure the convergence of algorithm. The video denoising results, refer to Figure 7, show that shared dictionary denoising works well on our DVS video. Different frames can be denoised without loss of structure of the objects in the frames.

\section{CONCLUSION}

This paper propose an improved approach for visualizing DVS with both high speed and rich information. The proposed method works well with DVS video and get good results. For the further work, we consider real-time DVS video denoising online, and set up the vehicle dataset of DVS.

\section{ACKNOWLEDGMENTS}

This work is supported by Natural Science Foundation (NSF) of China $(61472301,61632019)$ and Ministry of Education project (6141A02011601).

\section{REFERENCES}

[1] J. Huang, M. Guo, S. Chen. Live demonstration: A dynamic vision sensor with direct logarithmic output and full-frame picture-on-demand.In IEEE International Symposium on Circuits and Systems (ISCAS), pp. 456-456, 2016.

[2] Q.S. Jia, Z. Wen, L. Xia. Event-based sensor activation for indoor occupant distribution estimation.In International Conference on Control Automation Robotics \& Vision (ICARCV), pp. 240-245, 2012.

[3] H. Guo, J. Huang, M. Guo, S. Chen. Dynamic resolution event-based temporal contrast vision sensor. In IEEE International Symposium on Circuits and Systems (ISCAS),pp. 1422-1425, 2016.

[4] J.A. Pérez-Carrasco, B. Zhao, C. Serrano, B. Acha, T. Serrano-Gotarredona, S. Chen, and B. Linares-Barranco. Mapping from Frame-Driven to Frame-Free Event-Driven Vision Systems by Low-Rate Rate Coding and Coincidence Processing--Application to Feedforward ConvNets. IEEE transactions on pattern analysis and machine intelligence, vol. 35, no. 11, pp.2706-2719, 2013.

[5] F. Russo, G. Ramponi. A fuzzy filter for images corrupted by impulse noise. IEEE Signal Processing Letters, vol. 3, no.6, pp. 168-170, 1996

[6] I.Kubo, and S. Takenaka. Calculus on Gaussian white noise, I. Proceedings of the Japan Academy, Series A, Mathematical Sciences, vol. 56, no.8, pp. 376-380, 1980. 
[7] Z.Zhou, Z. Zhang, L. Shih, and W. Li. Column readout multiplexer for CMOS image sensors with multiple readout and fixed pattern noise cancellation.U.S. Patent No. 5,965,871. 12 Oct. 1999.

[8] F.N.Hooge. 1/f noise. Physica $B+C$, vol. 83, no.1, pp.14-23, 1976.

[9] R.E. Schild, B. Cholfin. CCD camera brightness monitoring of Q0957+ 561 A, B. The Astrophysical Journal, vol. 300, pp. 209-215,1986.

[10] M.A. Soto, J.A. Ramírez, L. Thévenaz. Image and video denoising for distributed optical fibre sensors. Proc. SPIE, Vol. 10323. No. EPFL-CONF-228211, 2017.

[11] H. Ji, C. Liu, Z. Shen, Y. Xu. Robust video denoising using low rank matrix completion. InIEEE Conference onComputer Vision and Pattern Recognition (CVPR),pp. 1791-1798, 2010.

[12] E.J. Balster, Y.F. Zheng, R.L. Ewing. Combined spatial and temporal domain wavelet shrinkage algorithm for video denoising. IEEE Transactions on Circuits and Systems for Video Technology, vol. 16, no. 2, pp. 220-230, 2006.

[13] Z. Li, W. Liu, M. Rong, and T. Liu. Implementation and evaluation of BM3D video denoising algorithms. Information Technology, vol. 4, pp.30-32, 2012.

[14] F.W. Edridge-Green. Persistence of vision. Nature, vol. 155 , pp. 178, 1945.

[15] M. Aharon, M. Elad, A. Bruckstein. \$ rm k \$-SVD: An algorithm for designing overcomplete dictionaries for sparse representation. IEEE Transactions on signal processing, vol. 54, no.11, pp. 4311-4322, 2006.

[16] X.Xie, J. Du, G. Shi, J. Yang, W. Liu,Wang Li. "DVS Image" Noise Removal using K-SVD Method. The Ninth International Conference on Graphic and Image Processing (ICGIP), 2017

[17] S. Gao, I.W.H. Tsang, Y. Ma. Learning category-specific dictionary and shared dictionary for fine-grained image categorization. IEEE Transactions on Image Processing, vol. 23, no.2, pp. 623-634, 2014. 